\title{
ON THE PROXIMAL RELATION IN TOPOLOGICAL DYNAMICS
}

\author{
JOSEPH AUSLANDER ${ }^{1}$
}

Let $(X, T)$ be a transformation group with compact Hausdorff phase space $X$. The points $x$ and $y$ of $X$ are said to be proximal provided, whenever $\beta$ is a member of the unique compatible uniformity of $X$, there exists $t \in T$ such that $(x t, y t) \in \beta$. If $x$ and $y$ are not proximal, they are said to be distal.

Let $P$ denote the proximal relation in $X . P$ is a reflexive, symmetric, $T$ invariant relation, but is not in general transitive or closed. As is customary, if $x \in X$, let $P(x)=[y \in X \mid(x, y) \in P]$.

If $x \in X$, the orbit of $x$ is the set $x T=[x t \mid t \in T]$. The closure of $x T$, denoted by $(x T)^{-}$is called orbit closure of $x$. A nonempty subset $M$ of $X$ is said to be a minimal orbit closure, or minimal set, if $M=(x T)^{-}$for all $x \in M$. If $A$ is a nonempty closed, $T$ invariant subset of $X$, then $A$ contains at least one minimal set $[3,2.22]$.

We may consider $T$ as a subset of $X^{x}$. (We identify two elements $t_{1}$ and $t_{2}$ of $T$ if $x t_{1}=x t_{2}$ for all $x \in X$.) Let $E$ be the closure of $T$ in $X^{X} . E$ is a compact semigroup (but not a topological semigroup); it is called the enveloping semigroup of $(X, T)$.

The enveloping semigroup of a transformation group was defined in [2]. Its algebraic properties, and their connection with the recursive properties of the transformation group are studied in [1].

A nonempty subset $I$ of $E$ is called a right ideal in $E$ if $I E C I$. If $I$ contains no proper nonempty subsets which are also right ideals, $I$ is called a minimal right ideal.

In Lemma 1, we summarize some results from [1] which we shall repeatedly use in this paper.

Lemma 1. (i) If $K$ is a closed right ideal in $E, K$ contains a minimal right ideal $I$.

(ii) If $I$ is a minimal right ideal in $E$, then $I$ is closed.

(iii) If $I$ is a minimal right ideal in $E$, and $x \in X$, then $x I$ is a minimal set in $X$.

(iv) If $K$ is a nonempty closed set in $E$ such that $K^{2} \subset K$, then $K$ contains an idempotent (i.e., an element $u$ such that $u^{2}=u$ ).

Presented to the Society, January 29, 1960 ; received by the editors December 22, 1959.

1 This work was supported in part by a National Science Foundation Research Grant. 
(v) If I is a minimal right ideal in $E$, $u$ an idempotent in $I$, and $p \in I$, then $u p=p$.

(vi) The points $x$ and $y$ of $X$ are proximal if and only if there exists a minimal right ideal $I$ in $E$ such that $x p=y p$ for all $p$ in $I$.

(vii) $P$ is an equivalence relation in $X$ if and only if $E$ contains exactly one minimal right ideal.

Observe that (i) tells us that $E$ always contains at least one minimal right ideal.

Lemma 2. Let $x \in X$, and let $M$ be a minimal set contained in $(x T)^{-}$. Then there exists a minimal right ideal $I$ in $E$ such that $M=x I$. Moreover, there is a point $y \in M$ such that $x$ and $y$ are proximal.

Proof. Let $F=[p \in E \mid x p \in M]$. If $p \in F$ and $q \in E$, then $x p q$ $\in M q \subset M$, so $p q \in F$. Therefore, $F E \subset F$. Also, since $M$ is closed in $X, F$ is closed in $E$. That is, $F$ is a closed right ideal in $E$. By Lemma 1 (i), $F$ contains a minimal right ideal $I$. Then $x I \subset x F \subset M$. By Lemma 1 (iii), $x I$ is a minimal set in $X$. Consequently, $x I=M$.

Now, let $u$ be an idempotent in $I$. Then $y=x u \in x I=M$, and $y u=x u^{2}=x u$, so $x$ and $y$ are proximal.

THEOREM 1. Suppose that $P$ is an equivalence relation in $X$. Then

(i) $X=\mathrm{U}_{\alpha} N_{\alpha}$, where the $N_{\alpha}$ are pairwise disjoint, $N_{\alpha} T \subset N_{\alpha}$, and each $N_{\alpha}$ contains precisely one minimal set $M_{\alpha}$.

(ii) If $x \in N_{\alpha}$, then $x$ is proximal to a point $y \in M_{\alpha}$.

(iii) If $P$ is closed in $X \times X$, then the sets $N_{\alpha}$ are closed.

Proof. (i) Let $x \in X$. Since proximal is an equivalence relation, $E$ contains just one minimal right ideal by Lemma 1, (vii). Therefore, by Lemma $2,(x T)^{-}$contains just one minimal set.

Let $\left\{M_{\alpha}\right\}$ be the class of minimal sets in $X$. Let

$$
N_{\alpha}=\left[x \in X \mid(x T)^{-} \supset M_{\alpha}\right] .
$$

It now follows that the $N_{\alpha}$ are pairwise disjoint, and that their union is $X$. By definition, each $N_{\alpha}$ contains just one minimal set, namely $M_{\alpha}$. It is clear that $N_{\alpha} T \subset N_{\alpha}$.

(ii) This is an immediate consequence of Lemma 2, since $M_{\alpha}$ $C(x T)^{-}$whenever $x \in N_{\alpha}$.

(iii) Suppose that $P$ is closed. Let $\left\{x_{n} \mid n \in D\right\}$ be a net in $N_{\alpha}$, and suppose $x_{n} \rightarrow x$. Then there exists $y_{n} \in M_{\alpha}$ such that $\left(x_{n}, y_{n}\right) \in P$. By choosing an appropriate subnet if necessary, let $y_{n} \rightarrow y \in M_{\alpha}$. Since $P$ is closed $(x, y) \in P$. Therefore $M_{\alpha}=(y T)^{-} \subset(x T)^{-}$and $x \in N_{\alpha}$. Hence $N_{\alpha}$ is closed. 
The following example shows that if $P$ is not closed the sets $N_{\alpha}$ need not be closed. The space $X$ is a compact subset of the plane. $X$ is the union of the circles $C_{n},(n=0,1,2, \cdots)$ defined by $x^{2}+\left(y-1+n /\left(n^{2}+1\right)\right)^{2}=\left(1-n /\left(n^{2}+1\right)\right)^{2}$. A point $Q$ on $C_{n}$ will be given the coordinates $(\alpha, n)$ where $\alpha$ is the angle between 0 and $\pi$ formed by the positive $x$ axis and the chord from the origin to $Q$.

We define a homeomorphism $f$ of $X$ onto $X$ by $(\alpha, n) f$ $=(\alpha+(1 / n) \sin \alpha, n)$, if $n>0$. We define $(\alpha, 0) f=(\alpha, 0)$. The group $T$ consists of the positive and negative powers of $f$. It is easily shown that $P$ is an equivalence relation in $X$.

If $0 \leqq \alpha<\pi$, let $M_{\alpha}$ be the set consisting of the point $(\alpha, 0)$. Then the sets $M_{\alpha}$ are the minimal sets of $X$. If $0<\alpha<\pi, N_{\alpha}=M_{\alpha}$. However, $N_{0}=(0,0) \cup C_{1} \cup C_{2} \cup \ldots$, which is not closed.

Let $u$ and $v$ be idempotents in $E$. We write $u \sim v$ if $u v=u$ and $v u=v$. It is easy to see that $\sim$ is an equivalence relation. In [1] it is proved that if $I_{1}$ and $I_{2}$ are minimal right ideals in $E$, and $u$ is an idempotent in $I_{1}$, then there is precisely one idempotent $u_{2}$ in $I_{2}$ such that $u_{1} \sim u_{2}$.

Let $(X, T)$ and $(Y, T)$ be transformation groups with the same phase group $T$. A homomorphism of $(X, T)$ onto $(Y, T)$ is a continuous map $\phi$ of $X$ onto $Y$ such that $(x t) \phi=(x \phi) t$ for all $x \in X$ and all $t \in T$.

Let $E(X)$ and $E(Y)$ be the enveloping semigroups of the transformation groups $(X, T)$ and $(Y, T)$ respectively. It is proved in [2] that if $\phi$ is a homomorphism of $(X, T)$ onto $(Y, T)$, then the map $\theta: E(X) \rightarrow E(Y)$ defined by $(x \phi)(p \theta)=(x p) \phi(x \in X, p \in E(X))$ is a continuous onto semigroup homomorphism.

Let $\left(\left(X_{i}, T\right) \mid i \in g\right)$ be a family of transformation groups, with the same phase group $T$. Let $X=X_{i \in g} X_{i}$, and let $x=\left(x_{i} \mid i \in g\right) \in X$. If $t \in T$ define $x t=\left(x_{i} t \mid i \in g\right)$. With this definition of $x t,(X, T)$ is a transformation group.

THEOREM 2. Let $\left(\left(X_{i}, T\right) i \in g\right)$ be a family of transformation groups with compact Hausdorff phase space, and the same phase group $T$. Let $X=\times_{i \in g} X_{i}$. Then proximal is an equivalence relation in $X$ if and only if proximal is an equivalence relation in each $X_{i}$.

Proof. Suppose proximal is an equivalence relation in each $X_{i}$. Let $\pi_{i}$ be the projection of $X$ onto $X_{i}$. Then $\pi_{i}$ is a homomorphism of $(X, T)$ onto $\left(X_{i}, T\right)$. Let $\theta_{i}$ be the induced homomorphism of $E(X)$ onto $E\left(X_{i}\right)$.

Now suppose that $I$ and $I^{\prime}$ are distinct minimal right ideals in $E(X)$. Then there are idempotents $u \in I$ and $u^{\prime} \in I^{\prime}$ with $u \sim u^{\prime}$. Then $u \theta_{i}$ and $u^{\prime} \theta_{i}$ are idempotents in $I \theta_{i}$ and $I^{\prime} \theta_{i}$ respectively such that $u \theta_{i} \sim u^{\prime} \theta_{i}$. Since proximal is an equivalence relation in $X_{i}, I \theta_{i}=I^{\prime} \theta_{i}$ 
and therefore $u \theta_{i}=u^{\prime} \theta_{i}$. Then, if $x \in X,(x u) \pi_{i}=\left(x \pi_{i}\right)\left(u \theta_{i}\right)=\left(x \pi_{i}\right)\left(u^{\prime} \theta_{i}\right)$ $=\left(x u^{\prime}\right) \pi_{i}$. This is true for every $i \in g$. That is $x u=x u^{\prime}$, and since $x$ is arbitrary $u=u^{\prime}$. But then $I \cap I^{\prime} \neq \varnothing$. Therefore, $E(X)$ has only one minimal right ideal, and proximal is an equivalence relation in $X$.

Now suppose proximal is an equivalence relation in $X$. Let $j \in \mathfrak{g}$, and suppose proximal is not an equivalence relation in $X_{j}$. Let $I_{j}$ and $I_{j}^{\prime}$ be distinct minimal right ideals in $E\left(X_{j}\right)$. Since $I_{j}$ and $I_{j}^{\prime}$ are closed and disjoint, $I_{j} \theta_{j}^{-1}$ and $I_{j}^{\prime} \theta_{j}^{-1}$ are closed disjoint right ideals in $E(X)$. Hence they contain minimal right ideals $I$ and $I^{\prime}$. This is a contradiction.

Let $\mathcal{P}$ be a property of a transformation group such that

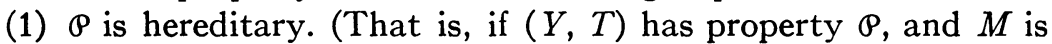
an invariant subset of $Y$, then $(M, T)$ has property $\left.P_{\text {. }}\right)$

(2) $P$ is productive. (That is, if $\left(\left(X_{i}, T\right) \mid i \in g\right)$ is a family of transformation groups, each of which has property $\mathcal{P}$, then $\left(X_{i \in \mathcal{I}} X_{i}, T\right)$ has property $P$.)

Then, if $(X, T)$ is any transformation group, there exists a smallest closed, $T$ invariant equivalence relation $R$ in $X$ such that the transformation group $(X / R, T)$ has property $\odot$ [2, Remark 8].

Let $P$ denote the property "proximal is an equivalence relation." Obviously $P$ is hereditary, and Theorem 2 tells us that $P$ is productive. Hence, we may always divide out appropriately so that in the quotient transformation group proximal is an equivalence relation.

If $p \in E$ and $z=(x, y) \in X \times X$, we define $z p=(x p, y p)$. With this definition, we may consider $p$ as a member of the enveloping semigroup of $X \times X$. If $R \subset X \times X$ and $H \subset E$ then $R H$ is the set $[z p \mid z \in R, p \in H]$, where $z p$ is defined as above.

The diagonal of $X \times X$, that is, the set $[(x, x) \mid x \in X]$ will be denoted by $\Delta$.

Observe that if $z=(x, y) \in X \times X$, then $z \in P$ if and only if $(z T)^{-} \cap \Delta$ $\neq \varnothing$.

THEOREM 3. The following are equivalent.

(i) $P$ is an equivalence relation in $X$.

(ii) Every orbit closure in $(X \times X, T)$ contains precisely one minimal set. $^{2}$

(iii) $P E \subset P$.

(iv) $P L \subset P$, where $L=[U I \mid I$ a minimal right ideal in $E]$.

(v) $P L \subset \Delta$.

Proof. We show that each statement in the theorem implies the following one and that (v) implies (i).

2 This condition was suggested to me by Professor Robert Ellis. 
Proof. If (i) holds, then by Theorem 2, proximal is an equivalence relation in $X \times X$. By Theorem 1 (i) every orbit closure in $X \times X$ contains precisely one minimal set.

Suppose (ii) holds. Let $z=(x, y) \in P$, and let $q \in E$. We show that $z q \in P$. By the remark above, it is sufficient to show that $(z q T)^{-} \cap \Delta$ $\neq \varnothing$. Let $M$ be a minimal set contained in $(z q T)^{-}$. Since $z q \in(z T)^{-}$, $(z q T)^{-} \subset(z T)^{-}$and therefore $M \subset(z T)^{-}$. Now $z \in P$, so $(z T)^{-} \cap \Delta \neq \varnothing$. Since $(z T)^{-} \cap \Delta$ is a nonempty closed $T$ invariant set, it contains a minimal set $M^{\prime}$. By (ii) $(z T)^{-}$contains just one minimal set, so $M=M^{\prime}$. Therefore, $M^{\prime} \subset(z q T)^{-}$and $(z q T)^{-} \cap \Delta \neq \varnothing$.

That (iii) implies (iv) is obvious.

Suppose (iv) is true. Let $z=(x, y) \in P$ and let $q \in L$. Then $q$ is in some minimal right ideal $I$. By (iv), $z q=(x q, y q) \in P$. That is, $x q$ is proximal to $y q$. By Lemma 1 (vi), there is a minimal right ideal $I^{\prime}$ in $E$ such that $x q r=y q r$, for all $r \in I^{\prime}$. Now $q I^{\prime}$ is a right ideal, and $q I^{\prime} \subset I E \subset I$, so we must have $q I^{\prime}=I$. Hence there is an $r \in I^{\prime}$ such that $q r=q$. Therefore, $x q=y q$, and $z q=(x q, y q) \in \Delta$. Therefore, $P L \subset \Delta$.

Finally, suppose (v) holds. Let $(x, y) \in P$ and $(y, z) \in P$. It follows from (v) that $x q=z q$ for all $q \in L$, so $(x, z) \in P$.

Corollary 1. If $P$ is closed in $X \times X$, then $P$ is an equivalence relation.

Proof. Let $z=(x, y) \in P$, and let $q \in E$. Let $\left\{t_{n} \mid n \in D\right\}$ be a net in $T$ such that $t_{n} \rightarrow q$. Now $\left(x t_{n}, y t_{n}\right) \in P$, and since $P$ is closed, $z q$ $=(x q, y q) \in P$. Therefore, (iii) of Theorem 3 is satisfied.

The example given above shows that the converse of Corollary 1 is not true. For example, the points $(\pi / 2, n)$ and $(0,0)(n=1,2, \cdots)$, are proximal. But $(\pi / 2, n) \rightarrow(\pi / 2,0)$ as $n \rightarrow \infty$ and $(\pi / 2,0)$ is clearly not proximal to $(0,0)$ since both of these points are mapped into themselves by $f$.

If $X$ is minimal under $T$, it is not known if $P$ must be closed when $P$ is an equivalence relation.

If $I$ is a minimal right ideal in $E$, let $J(I)$ denote the set of idempotents in $I$. Let

$$
J=U[J(I) \mid I \text { a minimal right ideal }] .
$$

The transformation group $(X, T)$ is said to be pointwise almost periodic if, for every $x \in X,(x T)^{-}$is a minimal set.

Theorem 4. Suppose $(X, T)$ is pointwise almost periodic, and let $x \in X$. Then

(i) $P(x)=x J$. 
(ii) Let I be a minimal right ideal in $E$. The points $x J(I)$ are mutually proximal. If $y$ is proximal to all $x^{\prime} \in x J(I)$, then $y \in x J(I)$. (That is, the sets $x J(I)$ are "maximal" sets of mutually proximal points.)

(iii) Let I be a minimal right ideal in $E$. Let $q \in I$ such that $(x, x q)$ $\in P$ for all $x \in X$. Then $q \in J(I)$.

Proof. (i) It is clear that $x J \subset P(x)$. Now, suppose $y \in P(x)$. Then there is a minimal right ideal $I$ in $E$ such that $x r=y r$, for all $r \in I$. Let $F=[r \in I \mid y r=y]$. Since $(y T)^{-}$is minimal, $(y T)^{-}=y I$, and $F \neq \varnothing$. Now $F$ is closed, and $F^{2} \subset F$. Therefore, $F$ contains an idempotent $u$. That is, there is a $u \in J(I)$ such that $y u=y$. But $x u=y u=y$, so $y \in x J(I)$.

(ii) Suppose $u, v \in J(I)$. By Lemma 1 (v), $u v=v$. Therefore, $x u v$ $=x v=x v^{2}=x v v$, so $(x u, x v) \in P$.

If $\left(y, x^{\prime}\right) \in P$, for all $x^{\prime} \in x J(I)$, then in particular $(y, x) \in P$. Therefore, by (i), there exists a minimal right ideal $I^{\prime}$ in $E$ and a $u^{\prime} \in J\left(I^{\prime}\right)$ such that $y=x u^{\prime}$. Let $u \in J(I)$ such that $u \sim u^{\prime}$. By hypothesis, $\left(x u^{\prime}, x u\right)=(y, x u) \in P$. Then, for some minimal right ideal $I^{\prime \prime}$ in $E$, $x u^{\prime} p^{\prime \prime}=x u p^{\prime \prime}$ for all $p^{\prime \prime} \in I^{\prime \prime}$. Let $u^{\prime \prime} \in J\left(I^{\prime \prime}\right)$ such that $u \sim u^{\prime} \sim u^{\prime \prime}$. Then $x u^{\prime} u^{\prime \prime}=x u u^{\prime \prime}$. But $u^{\prime} u^{\prime \prime}=u^{\prime}$, and $u u^{\prime \prime}=u$, so $y=x u^{\prime}=x u$ $\in x J(I)$.

(iii) Note that for all $x \in X$, and all $u \in J(I),(x u, x q) \in P$. For by hypothesis, $(x u, x u q) \in P$. By Lemma $1(\mathrm{v}), u q=q$, so $x u q=x q$.

Now, fix $x \in X$ and let $y=x q$. Then $y$ is proximal to $x u$, for all $u \in J(I)$. Hence, by (ii) $x q=y=x v$, where $v \in J(I)$ and $v$ depends (apparently) on $x$.

Now, by $[1$, Lemma $2(3)]$ there is a $w \in J(I)$ such that $q w=q$. Since $v w=w$, we have $x q=x q w=x v w=x w$. That is, for all $x \in X$, $x q=x w$, so $q=w \in J(I)$.

\section{REFERENCES}

1. Robert Ellis, $A$ semigroup associated with a transformation group, Trans. Amer. Math. Soc. vol. 94 (1960) pp. 272-281.

2. Robert Ellis and W. H. Gottschalk, Homomorphisms of transformation groups, Trans. Amer. Math. Soc. vol. 94 (1960) pp. 258-271.

3. W. H. Gottschalk and G. A. Hedlund, Topological dynamics, Amer. Math. Soc. Colloquium Publications, vol. 36, 1955.

Carnegie Institute of Technology 